\title{
jfml
}

Vol 2 (2019), No 2: 28-29

DOI: $10.21248 / \mathrm{jfml} .2019 .43$

\section{Mobile Medienpraktiken im Spannungsfeld von Öffentlichkeit, Privatheit und Anonymität}

Die Beiträge des Themenhefts

Katharina König \& Florence Oloff

Die in dem Themenheft versammelten Beiträge setzen sich unter verschiedenen Fragestellungen, im Rahmen unterschiedlicher methodischer Ansätze und jeweils eigener Datensets mit Öffentlichkeit, Privatheit und Anonymität im kommunikativen Handeln mit mobilen Medien auseinander.

Der Beitrag „Narratives 2.0. A Multi-Dimensional Approach to Semi-Public Storytelling in WhatsApp Voice Messages“ von Katharina König analysiert, wie NutzerInnen Erzählungen in Sprachnachrichten dazu einsetzen, in der ,kontrollierten' Öffentlichkeit von WhatsApp-Gruppenchats Privatheit herzustellen. Die Gruppenmitglieder unterstützen diese Rahmung durch zumeist gleichförmig gestaltete affiliative Textnachrichten. Um die Charakteristika des Erzählens in dem auf einen kontinuierlichen Dialog ausgelegten mobilen Messenger-Chat adäquat fassen zu können, sollten die Multimodalität und die Sequenzierung in Einzelpostings bei der Analyse mit in den Blick genommen werden.

In ihrer ethnographischen Studie „Smartphone-Based Language Practices among Refugees: Mediational Repertoires in Two Families“ befassen sich Olga Artamonova und Jannis Androutsopoulos mit der Nutzung mobiler Medien in einer syrischen und einer afghanischen Flüchtlingsfamilie in Hamburg. Mit dem in dem Beitrag entwickelten Konzept der mediational repertoires nehmen sie intraund interfamiliäre Unterschiede bei Praktiken der multimodalen und multilingualen Smartphone-Kommunikation in den Blick. Zugleich können die AutorInnen nachzeichnen, welche zentrale Rolle das Smartphone bei der Herstellung verschiedener transnationaler vernetzter Öffentlichkeiten und beim informellen bzw. selbstgesteuerten Erwerb der deutschen Sprache spielt.

David Suderland untersucht in „oh isch FIND_s nich;'. Eine konversationsanalytische Untersuchung sprachlicher Bezugnahmen 
auf smartphone-gestützte Suchanfragen in Alltagsgesprächen“, wie GesprächsteilnehmerInnen - in Vorbereitung einer gemeinsamen Betrachtung auf dem Mobilgerät - mediale Inhalte suchen und finden. Anhand von Audioaufnahmen deutschsprachiger Alltagsgespräche stellt der Autor kollaborative Suchen sowie suchbegleitende Kommentierungen vor. Diese sprachlichen Praktiken machen die ursprünglich intransparente Smartphonebenutzung öffentlich zugänglich und ermöglichen es anderen, den Fortschritt der Suchaktivität nachzuvollziehen oder diese sogar aktiv mitzugestalten.

In einem Kontext, in dem das Smartphone als Medium der Selbstpräsentation dient und in dem zugleich die Zusammensetzung der rezipierenden Publika unbekannt ist, spielen Praktiken der Anonymisierung eine zunehmend wichtigere Rolle. In dem Beitrag „Creating a Self-Image. Face-Work and Identity Construction Online“ zeigen Saskia Kersten und Netaya Lotze auf, wie sich die Selbstwahl von Online-Nicknames bei Plattformen wie Twitter und Flickr in einem Spannungsfeld von Authentizität und Anonymität bewegt. NutzerInnen bedienen sich verschiedener Verfahren der Namensgebung, um zwischen dem sozialen Bedürfnis der Vernetzung mit anderen einerseits und dem Wunsch nach Privatsphäre andererseits navigieren zu können, und stellen so spezifische Identitätspositionen für ihr Handeln in sozialen Medien her.

In seiner experimentellen, designorientierten Studie „Face to Face. Sozio-interaktive Potentiale der Videotelefonie“ interessiert sich Tobias Held für den Zusammenhang zwischen bestimmten Endgeräten (bzw. deren Verwendungen) und empfundener Intimität im Rahmen von Videotelefonaten. Hier wird illustriert, wie StudienteilnehmerInnen die empfundene Nähe zum/zur KommunikationspartnerIn in Hinblick beispielsweise auf verschiedene Bildformate, Bildausschnitte oder Blickwinkel auf das Gerät beurteilen. Basierend auf dieser Studie sowie einer vorangegangenen theoretischen Diskussion schlussfolgert der Autor, dass spezifische visuelle Einstellungen des Endgerätes bei Videotelefonaten für die Herstellung von kommunikativer Verbundenheit und Nähe besser geeignet sind.

Florence Oloff setzt sich in ihrem das Themenheft abschließenden Beitrag „Some Systematic Aspects of Self-Initiated Mobile Device Use in Face-to-Face Encounters“ mit verschiedenen Formen selbst-initiierter Smartphone-Benutzung in videografierten tschechischen Alltagsgesprächen auseinander. Mithilfe eines konversationsanalytischen und multimodalen Ansatzes wird hier untersucht, wie InteraktionsteilnehmerInnen die Nutzung ihres Mobilgeräts (beispielsweise zum Schreiben einer Textnachricht) hör- und sichtbar öffentlich rahmen. Individuelle Mobilgerätnutzung in Anwesenheit anderer ist nicht grundsätzlich problematisch, sondern wird situationsabhängig durch handlungsbeschreibende Ankündigungen sowie spezifische Antworten darauf öffentlich verhandelt. 